\title{
Autorregulación para el aprendizaje en estudiantes universitarios*
}

DOI: http://dx.doi.org/10.17081/eduhum.18.31.1382

\author{
Marbel Gravini-Donado, \\ orcid.org/0000-0003-3972-5271 \\ Myriam Esther Ortiz-Padilla ${ }^{2}$ \\ orcid.org/0000-0001-8964-9428 \\ Lilia Campo-Ternera ${ }^{3}$ \\ orcid.org/0000-0002-1472-0362 \\ Universidad Simón Bolivar, Colombia
}

Recibido: 26 de febrero de 2016

Aceptado: 5 de agosto de 2016

\section{Learning autoregulation for university students}

Palabras clave:

Autorregulación,

Motivación hacia el aprendizaje, Estrategias de aprendizaje,

Estrategia de manejo de recursos.

Keywords:

Auto regulation,

Learning motivation,

Learning strategies,

Managing resources strategy.

\begin{abstract}
Resumen
En este artículo se presentan los resultados de una investigación descriptiva realizada con estudiantes de tercer semestre de los programas académicos de una universidad privada, cuyo objetivo consistió en describir su autorregulación para el aprendizaje. La muestra fue de 130 estudiantes escogidos con un muestreo aleatorio estratificado de una población de 370 de ellos. El instrumento utilizado fue el MSLQ (Motivated Strategies for Learning Questionnaire) diseñado por Pintrich, Smith, García y McKeachie (1991) traducido al español. Los resultados generales indican que los estudiantes en su mayoría poseen niveles por encima de la media en sus estrategias metacognitivas, aunque se encuentran algunas diferencias por programas. En el caso del Programa de Medicina, se observa que obtiene las puntuaciones más altas en casi todas las dimensiones de autorregulación evaluadas; sin embargo, en estos estudiantes se encontraron niveles más altos de ansiedad con respecto a los otros programas.
\end{abstract}

\begin{abstract}
This article presents the results of a descriptive research work with Third semester students of different academic programs of a private university, whose main objective consisted in describing its learning auto regulation. The sample includes 130 students chosen at random stratified from a population of 370 . The instrument used was the MSLQ (MotivatedStrategiesforLearningQuestionnaire) designed by Pintrich, Smith, García y McKeachie (1991) translated into Spanish. The results indicate that students in its majority have metacognositive strategy levels over the media, although there are some program differences. In the case of Medicine, it has been observed that the highest grading were for nearly all the dimensions of evaluated auto regulation, however, in these students higher levels of anxiety were found in relation to other programs.
\end{abstract}

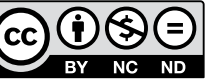

Referencia de este artículo(APA): Gravini-Donado, M., Ortiz-Padilla, M.\&Campo-Ternera,L.(2016). Autorregulación para el aprendizaje en estudiantes universitarios. Revista Educación y Humanismo, 18(31), 326-342. http://dx.doi.org/10.17081/eduhum.18.31.1382

* Esta investigación se desarrolló con la participación de las estudiantes del programa de Psicología: María Fernanda Cuentas, Andrea Gómez Serna y Reyna Flórez, de la Universidad Simón Bolívar.

1. Magíster en Educación. Psicóloga. Líder del Grupo Sinapsis Educativa y Social, Universidad Simón Bolívar. Email: mgravini1@unisimonbolivar.edu.co

2. Magíster en Educación. Psicóloga. Docente del Instituto Nacional de Formación Técnica Profesional (INFOTEP). Investigadora Grupo Psicología Educativa. Email: mortiz@unisimonbolivar.edu.co

3. Doctorante en Administración. Magíster en Psicología. Profesora investigadora, Coordinadora del programa Ondas. Universidad Simón Bolívar. Email: licampo@unisimonbolivar.edu.co 


\section{Introducción}

Con el pasar de los años la educación ha tomado otro enfoque en términos de conocimiento, puesto que en épocas anteriores se creía que el estudiante solo era un punto de recepción de información. A lo largo del tiempo se ha podido ver cómo el alumno busca mediante diferentes procesos, no solo la recepción de dicho conocimiento, sino también su utilidad ante situaciones del medio. Se ha observado el acercamiento, autoconocimiento y utilidad individual por parte de los estudiantes ante diversas tareas, que muestran así de qué manera ellos tienen capacidad de autorregular su propio aprendizaje, es decir, planificar cuáles estrategias han de utilizar en cada situación, aplicarlas, controlar el proceso, evaluarlo para detectar posibles fallos, y, en consecuencia, transferir todo ello a una sola actuación (Limón, 2004; Sinatra, 2004).

Igualmente se constata que las universidades han venido implementando sistemas tutoriales para estudiantes de nuevo acceso, con el fin de potenciar su aprendizaje en especial en el desarrollo de conocimientos y competencias relacionadas con la escritura, el lenguaje académico y el aprendizaje autorregulado (García Ros \& Pérez González, 2011).

Pintrich y Zusho (2002, citado en Núñez, Solano, Gonzales \& Rosario, 2006) expresan que aquellos estudiantes que son capaces de regular su propio aprendizaje, pese a los distractores presentes en un aula de clases, obtendrán mejores resultados y su proceso de aprendizaje será más efectivo.
A partir de estas concepciones ha nacido un interés en estudiar esta temática en personas de diferentes edades, sexos y contextos, así como de su influencia en diferentes aspectos: la comunicación, espacios organizacionales y ambientes educativos conforme es el caso de este artículo. Los antecedentes de este estudio se encuentran en trabajos como el de Escorcia (2010), que refiere acerca de los conocimientos metacognitivos y autorregulación de estudiantes universitarios en la producción de textos escritos, teniendo como objetivo investigar la problemática mundial de deserción de los estudiantes universitarios y analizar en particular las competencias metacognitivas de este público en el campo de la redacción. Se concluye que, a pesar de poseer informaciones pertinentes para la tarea, los estudiantes se quedan en la modalidad descriptiva del discurso $\mathrm{y}$ sin estrategias que tengan en cuenta las expectativas del destinatario. En general, centran sus procesos de redacción en la transcripción de ideas con poco control consciente de la planeación y de la revisión de los textos.

Otros investigadores interesados en este tema son Valle, Cabanch, Rodríguez, Núñez y González (2006), quienes hicieron un proyecto sobre Metas académicas, estrategias cognitivas y estrategias de autorregulación del estudio. Su objetivo era analizar las diferencias en estrategias cognitivas y en estrategias de autorregulación del estudio según los niveles de las metas académicas. Los resultados muestran que, además de las metas orientadas al aprendizaje, hay otras metas (orientadas a la valoración social y a la 
consecución de una buena situación laboral en el futuro) que promueven el uso de estrategias y la implicación en el estudio. Incluso aquellas metas orientadas al yo, que llevan a una implicación en el estudio derivada de una defensa del yo, y las derivadas de una búsqueda de mejora de la autoestima también están relacionadas significativamente con el uso de estrategias cognitivas y de autorregulación del estudio.

Una de las obras más citadas y relevantes es la de los autores De la Fuente, Pichardo, Justicia y Berbén (2008), que investigaron acerca de los enfoques de aprendizaje, autorregulación y rendimiento en tres universidades europeas, centrándose en el estudio de la relación entre el enfoque de aprendizaje de los estudiantes universitarios con la forma de regular su aprendizaje y el tipo de rendimiento. Como resultado se observó una relación entre los enfoques de aprendizaje y la autorregulación que desarrollan los estudiantes de estas universidades, concretada en una mejor planificación y control de ejecución. Se comprueba también que un aprendizaje más profundo va acompañado de mayor planificación, de un comportamiento y estrategias más reguladoras.

Una investigación interesante fue la desarrollada por diferentes universidades europeas (Universidad de Murcia, Universidad Católica de San Antonio y Universidade Do Minho), las cuales considerando el Marco del Espacio Europeo de Educación Superior, adelantaron el Proyecto PACA (Promoción de Autorregulación y Competencias de Aprendizaje), en el que se diseñaron 13 cartas con el propósito de propiciar la reflexión entre los matriculados de primer semestre a partir de las experiencias que un estudiante con sentido del humor va narrando sobre los procesos de adaptación a la institución, los desafíos y las anécdotas de la vida universitaria, entre otros. Al evaluar el impacto del proyecto, el estudio encontró que los participantes logran mejorar su conocimiento declarativo de estrategias de aprendizaje; sin embargo, para optimizar sus estrategias de autorregulación es necesario que el estudiante, además de querer, sepa hacerlo; por eso es importante que aumente su conocimiento estratégico a fin de aplicarlo en una tarea (Hernández-Pina, Rosario, Cuesta Sáez de Tejada, Martínez-Clares \& Ruiz-Lara, 2006).

En otros trabajos se ha evidenciado que el rendimiento escolar, particularmente en Lengua y Matemáticas, puede ser explicado por el uso de estrategias de aprendizaje autorregulado y cómo este tipo de comportamiento deseable puede estar condicionado por la utilidad percibida de su uso y por la competencia percibida para su ejecución. Los resultados obtenidos muestran que a mayor uso de estrategias de autorregulación del proceso de aprendizaje, mayor es el rendimiento, y viceversa, y que el uso de dichas estrategias depende fuertemente de la utilidad percibida de tal comportamiento y de la autoeficacia para su uso efectivo. Estos datos sugieren la importancia de que los profesores utilizando estrategias de autorregulación en las tareas previstas en el currículo, las apliquen a situaciones concretas 
de aprendizaje y busquen así mismo su transferencia a otros contextos y tareas escolares, ya que esto hará más visible su utilidad, y la práctica generada es percibida como competencia en los alumnos (Rosário, Lourenço, Paiva, Núñez, González-Pienda \& Valle, 2012).

Los diversos trabajos e investigaciones sobre este tema han dejado avances en diferentes áreas y temáticas de la educación, con resultados notorios y de gran valor. Esos estudios han permitido ver cambios en el modelo tradicional de enseñanza unidireccional, que privilegiaba el conocimiento del maestro ante el alumno; este tenía solo el papel de receptor ante el docente, y utilizaba únicamente procesos de memoria con el fin de lograr un aprendizaje, dejando de lado la interacción o reciprocidad de conocimiento y aprendizaje entre ambos. En contraposición, en la actualidad el desarrollo de la autorregulación del pensamiento, o el aprendizaje autorregulado, considera al estudiante como un promotor metacognitivo, motivacional y comportamentalmente activo de su rendimiento académico que: a) está motivado porque se siente capaz de abordar las actividades de estudio; b) planifica su proceso de aprendizaje, lo controla y lo regula; c) desarrolla una conciencia clara de los resultados de su conducta; y d) es sensible y dispone de recursos para gestionar el entorno físico y social en el que se desarrolla su proceso de aprendizaje (Zimmerman, 1994).

Estas actitudes serán tenidas en cuenta como características personales, contextuales y el ren- dimiento real del estudiante, y a partir de tal caracterización básica debemos describir los componentes implicados en este tipo de aprendizaje entre los que se incluye la gestión activa de recursos cognitivos, contextuales y motivacionales, que se desarrollará en tres fases.

La primera fase, de planificación, se refiere a la parte de la autorregulación previa a la ejecución de las tareas y procesos desarrollados durante el aprendizaje, y en ellos entran en juego tanto expectativas y creencias motivacionales como conocimiento y estrategias cognitivas (McCombs, 1989), dada la interacción entre el sistema metacognitivo con el sistema cognitivo y el sistema afectivo, lo cual implicará tanto el conocimiento (conciencia) como el control (autorregulación) de la cognición y del afecto. La planificación estará especialmente afectada por las percepciones que el aprendiz tenga de los requisitos de la tarea y por los esquemas, conocimientos y estrategias de los que el sujeto dispone al abordar cada tarea de aprendizaje.

Por su parte, el control constituye una segunda fase de la autorregulación y contempla tanto la observación que realiza el aprendiz de la actividad llevada a cabo, y de las condiciones que la rodean, como los resultados obtenidos y el sostenimiento del esfuerzo y la persistencia en el proceso de aprendizaje. Finalmente, está la tercera fase de la autorregulación, la de valoración, que incorpora procesos de evaluación y explicación de los resultados obtenidos, así como los modos de reacción que provocan. 
El modelo de Pintrich (fases, áreas y procesos implicados en el aprendizaje autorregulado)

Pintrich fue el autor base durante este proceso de investigación, ya que él propuso un modelo como uno de los intentos de síntesis más importantes realizados sobre los diferentes procesos y actividades que ayudan a acrecentar la autorregulación del aprendizaje. Su propuesta está basada en una perspectiva sociocognitiva, con el objetivo de clasificar y analizar los distintos procesos que según la literatura científica están implicados en el aprendizaje autorregulado. En dicho modelo, los procesos reguladores se organizan en función de cuatro fases: a) la planificación, b) la autoobservación (self-monitoring), c) el control, y d) la evaluación. A su vez, dentro de cada una de ellas, las actividades de autorregulación se enmarcan dentro de cuatro áreas: la cognitiva, la motivacional/afectiva, la comportamental y la contextual.

Estas cuatro fases representan para Pintrich una secuencia general por donde el alumno avanza a medida que realiza la tarea, pero no están jerárquica o linealmente estructuradas. Según este autor, como tales fases pueden darse de forma simultánea y dinámica, se produce una múltiple interacción entre los diferentes procesos y componentes incluidos en ellas.

De esta manera los procesos autorreguladores se inician en la fase de planificación, donde se destacan como actividades importantes: el establecimiento de las metas que se desean alcanzar o el objetivo específico que se quiere lograr con la tarea, la activación del conocimiento previo sobre la materia y del conocimiento metacognitivo (darse cuenta de las dificultades que entrañan las diferentes tareas, identificar los conocimientos y habilidades necesarias para abordarlas, conocimiento acerca de los recursos y estrategias que les pueden ayudar a solucionar la tarea, etc.) -área cognitiva-; la activación de las creencias motivacionales (autoeficacia, metas, valor dado a la tarea, interés personal) y las emociones -área motivacional/afectiva-; la planificación del tiempo y del esfuerzo que se va a emplear en las tareas -área comportamental-y la activación de las percepciones respecto de la tarea y el contexto de clase -área contextual- (Torrano \& González, 2004).

Dentro de la fase de autoobservación (selfmonitoring) se incluyen las actividades que ayudan al alumno a tomar conciencia del estado de su cognición, su motivación, su afecto, su uso del tiempo y del esfuerzo, así como de las condiciones de la tarea y del contexto. Por ejemplo, allí se incluyen aquellas relacionadas con la autoobservación de la comprensión (toma de conciencia metacognitiva). Estas se manifiestan cuando los alumnos son conscientes de que no han entendido algo que acaban de leer o escuchar, cuando son conscientes de que están leyendo demasiado de prisa teniendo en cuenta la naturaleza del texto o sus metas, o cuando observan activamente su comprensión lectora, haciéndose preguntas a sí mismos para saber si lo han entendido (Pressley \& Afflerbach, 1995). 
Esta fase asimismo abarca los procesos que los alumnos ponen en marcha para ser conscientes de su patrón motivacional (si se sienten competentes para realizar las tareas, si las valoran o qué metas guían y dirigen su conducta académica), de su propio comportamiento ("Tengo que dedicar más tiempo y esfuerzo para comprender este capítulo", "Necesito buscar ayuda"), así como de las características de la tarea y del contexto del aula (qué reglas existen en clase, cómo se evaluará el rendimiento, los requerimientos de la tarea, los sistemas de recompensas y castigos, la actuación del profesor, etc.).

A la luz de los resultados de la fase anterior se ponen en marcha las actividades de control, que engloban la selección y utilización de estrategias para controlar el pensamiento (uso de estrategias cognitivas y metacognitivas), la motivación y el afecto (estrategias motivacionales y de control emocional), así como las relacionadas con la regulación del tiempo y del esfuerzo y con el control de las diversas tareas académicas, el clima y la estructura de la clase.

Por último, la fase de reflexión o evaluación abarca los juicios y las evaluaciones que los alumnos realizan acerca de su ejecución en la tarea, comparándola con los criterios previamente establecidos por ellos (o por el profesor); las atribuciones que realizan sobre las causas de sus éxitos o fracasos; las reacciones afectivas que experimentan ante los resultados, como consecuencia de las atribuciones realizadas; la elección del comportamiento que se llevará a cabo en el futuro, así como las evaluaciones generales sobre la tarea y el ambiente de clase. En este modelo se centró esta investigación.

Entre otros modelos de autorregulación está el modelo de aprendizaje "adaptable" de Boekaerts (1997), el cual considera que los estudiantes están intrínsecamente motivados a autorregular su actuación en términos de dos prioridades básicas: alcanzar metas de crecimiento o desarrollo personal (por ejemplo, incrementar sus habilidades sociales y cognitivas) y proteger su bienestar emocional dentro de unos mínimos razonables (por ejemplo, mostrarse ante los demás como seres capaces y proteger su ego).

Zimmerman (1995) plantea que los psicólogos educativos deben ampliar su mirada a los procesos de autorregulación y tener en cuenta una serie de factores que van más allá de la metacognición, como son: la motivación, las habilidades de automanejo de contingencias ambientales, la cognición social y creencias de autoeficacia, que pueden incidir en las dudas o fracasos que tienen al elegir o descartar una estrategia de autorregulación. También incluye el conocimiento y el sentido de control personal para ejecutar estas habilidades en contextos relevantes.

Gran parte de los autores coinciden en vincular a la motivación intrínseca con aquellas acciones realizadas por el interés que genera la propia actividad, considerada como un fin en sí misma y no como un medio para alcanzar otras metas. Tapia (1995) sugiere que la motivación 
parece incidir sobre la forma de pensar $y$, por lo tanto, sobre el aprendizaje. Es posible que el alumno motivado intrínsecamente esté más dispuesto a aplicar un esfuerzo mental significativo durante la realización de la tarea, a comprometerse en procesamientos más ricos y elaborados y en el empleo de estrategias de aprendizaje más profundas y efectivas. En cambio, parece más probable que un estudiante motivado extrínsecamente se comprometa en ciertas actividades solo cuando estas ofrecen la posibilidad de obtener recompensas externas; además, es posible que tales estudiantes opten por tareas más fáciles, cuya solución les asegure la obtención de la recompensa.

Similarmente apareció Albert Bandura (1982), con su teoría sobre la autoeficacia. En general, la autoeficacia tiene que ver con los juicios que realizan las personas sobre sus propias capacidades para lograr organizar y ejecutar las acciones necesarias a fin de alcanzar los resultados que pretenden. La autoeficacia incluye la ejecución y organización de cursos de acción, por lo que presenta una perspectiva situacional de la competencia, ya que incluye no solo el conocimiento, sino también los juicios sobre las estrategias, así como los modos de regulación necesarios para desarrollar una tarea.

En una investigación de Wood y Bandura (1989) sobre el impacto de las concepciones sobre la capacidad de mecanismos de autorregulación y toma de decisiones complejas, los autores proporcionaron evidencias que apoyan la con- cepción de que las personas que deben tomar decisiones complejas tienen esta habilidad gracias a los mecanismos de autorregulación con los que logran su rendimiento. El concepto de poder adquirir dicha habilidad fomenta un sentido alto de eficacia personal. Estos resultados son acordes con numerosas investigaciones que concluyen que los logros humanos requieren un fuerte sentido de eficacia y autorregulación personal.

\section{Método}

Diseño: El estudio realizado es de corte cuantitativo desde un enfoque empírico analítico. Para lograr los objetivos el diseño utilizado fue descriptivo de corte transversal.

Participantes: La población de referencia estuvo conformada por 370 estudiantes de tercer semestre diurno y nocturno de las diferentes facultades de una universidad privada de la ciudad de Barranquilla, Colombia. Se tomó como población un programa por cada una de las áreas de conocimiento que ofrece esta Alma mater. El muestreo utilizado para escoger la muestra fue aleatorio estratificado, y esta quedó constituida de la siguiente manera: 29 estudiantes de Medicina, 27 estudiantes de Ingeniería Industrial, 47 estudiantes de Derecho y 27 estudiantes de Administración de Empresas. Por tal razón, el tamaño de la muestra se ajustó a 130 estudiantes.

Instrumentos: El instrumento utilizado fue el MSLQ (Motivated Strategies for Learning Questionnaire) diseñado por Pintrich, Smith, García y McKeachie (1991) y traducido al es- 
pañol para esta investigación. El MSLQ fue diseñado para ser usado por los investigadores como un instrumento de medida para investigar la naturaleza de la motivación del estudiante y el uso de estrategias de aprendizaje, y por los instructores y los estudiantes como un medio de evaluar la motivación de los estudiantes y las habilidades de estudio dentro de un curso determinado. La puntuación alcanzada con esta prueba indica el nivel de aprendizaje autorregulado del estudiante así: una puntuación mayor de 75 evidencia un nivel alto, entre 51 y 74 se presenta un nivel medio, de 26 a 50 el nivel obtenido es bajo y si la puntuación es menor a 25 , el resultado se califica como inferior.

Para la traducción y validación de la versión original en inglés del cuestionario Motivated Strategies for Learning Questionnaire, diseñado por Pintrich, Smith, García y McKeachie (1991), se conformó un equipo de trabajo integrado por dos personas bilingües con lengua española de origen y por investigadores, pues luego del trabajo inicial, el cuestionario fue sometido a discusión, hasta lograr un consenso sobre la primera versión del texto en español. Posteriormente se realizó un debriefing con la primera versión en español del cuestionario en busca de los siguientes objetivos: identificar la comprensión de palabras o términos por parte de los sujetos, así como las dudas generadas para responder los ítems, evaluar las opciones de respuestas cerradas y la sensibilidad de las preguntas, y, por último, examinar las sugerencias en la redacción de las preguntas propuestas por los sujetos. Finalmente, el equipo conformado realiza la validación de contenido y los ajustes necesarios que dan lugar a la versión final en idioma español del instrumento.

\section{Resultados}

A continuación, por medio de gráficas estadísticas, se presentan los resultados obtenidos luego de la evaluación realizada a la muestra seleccionada. En la Figura 1 se muestran inicialmente los resultados de cada uno de los programas comparando entre sí sus puntaciones obtenidas en cada una de las subescalas y en las escalas. Se mostrarán, también, los resultados correspondientes a la escala de ansiedad y, por último, se expondrán los resultados de cada uno de los programas hallados a nivel general.

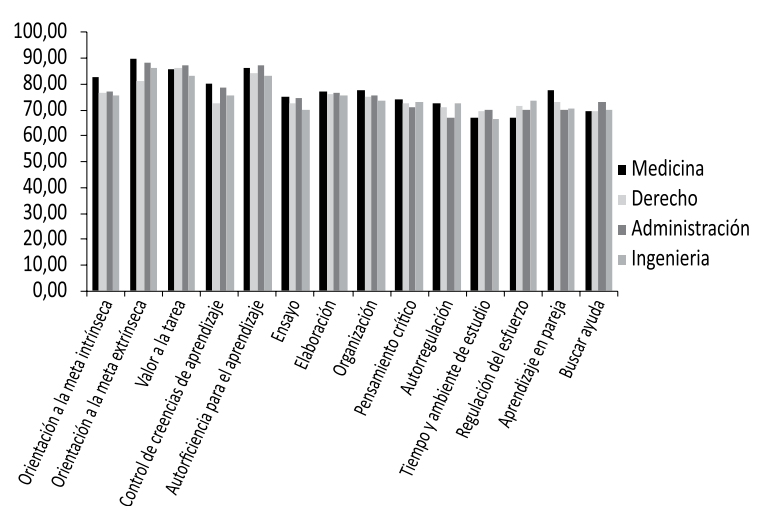

Figura 1. Resultados de las puntuaciones obtenidas por los estudiantes de los programas participantes en las subescalas del cuestionario

Fuente: Resultados de investigación

Luego de evaluar los programas de Medicina, Ingeniería Industrial, Derecho y Administración de Empresas, en la subescala de orientación a la meta intrínseca los resultados indican que el Programa de Medicina muestra una puntuación de $82,64 \%$, superior en comparación con los 
demás programas; mientras que el Programa de Ingeniera Industrial mostró un menor puntaje en esta subescala con un $75,26 \%$.

En la subescala de orientación a la meta extrínseca, el Programa de Medicina mostró una puntuación de 89,90 \%, mientras que el Programa de Derecho evidenció un puntaje de 81,23 \%, inferior a los otros programas evaluados.

En la subescala de valor a la tarea, el Programa de Administración de Empresas mostró un puntaje superior de $87,21 \%$, mientras que el Programa de Ingeniería Industrial puntuó un porcentaje menor de $82,98 \%$ con relación a los demás programas.

En la subescala de control de creencias de aprendizaje, el Programa de Medicina mostró un porcentaje mayor de $80,17 \%$, en comparación con los demás programas, mientras el Programa de Derecho obtuvo un porcentaje menor de $72,64 \%$.

En la subescala de autoeficiencia para el aprendizaje, el Programa de Medicina obtuvo un porcentaje superior de $86,15 \%$ en comparación con los otros programas, y el programa con el menor puntaje fue Ingeniería Industrial con $83,4 \%$.

En la subescala ensayo, el programa con puntuación mayor es Medicina, con un 75 \%, mientras que Ingeniería Industrial es el programa con el porcentaje menor: $70,11 \%$.
En la subescala de elaboración, el Programa de Medicina mostró un porcentaje mayor de 76,93 \% en comparación con el Programa de Ingeniera Industrial que obtuvo una puntuación menor de $75,57 \%$.

En la subescala de organización, el Programa de Medicina mostró un puntaje superior de $77,83 \%$ en comparación a los demás programas, mientras el Programa de Ingeniería Industrial obtuvo un 73,41\%, menor a los demás programas.

En la subescala de pensamiento crítico, el Programa de Medicina obtuvo un porcentaje mayor en comparación con los demás programas de $73,89 \%$, mientras que el Programa de Administración de Empresas obtuvo el menor porcentaje: $70,90 \%$.

En la subescala de autorregulación, el Programa de Medicina obtuvo mayor porcentaje de $72,62 \%$, mientras que el menor porcentaje lo obtuvo el Programa de Administración de Empresas con un resultado de $67,20 \%$.

La subescala de tiempo y ambiente de estudio muestra que el programa con mayor porcentaje es Administración de Empresas, con 69,91 \%, mientras que el Programa de Ingeniería Industrial obtuvo un puntaje menor de $66,60 \%$ en comparación con los demás programas.

La subescala de regulación del esfuerzo muestra que el Programa de Ingeniería Indus- 
trial, obtuvo un puntaje superior de 73,54\%, mientras que el Programa de Medicina obtuvo un $66,87 \%$, lo cual es menor a lo que obtuvieron los demás programas evaluados.

La subescala aprendizaje en pareja muestra que el Programa de Medicina obtuvo un puntaje superior de 77,83 \% en comparación con el Programa de Administración de Empresas, con un puntaje de $69,84 \%$, menor al obtenido por los demás programas.

En la subescala de buscar ayuda, el programa con mayor puntaje es Administración de Empresas con un $73,02 \%$, mientras que el Programa de Derecho obtuvo el menor puntaje: 69,53\%.

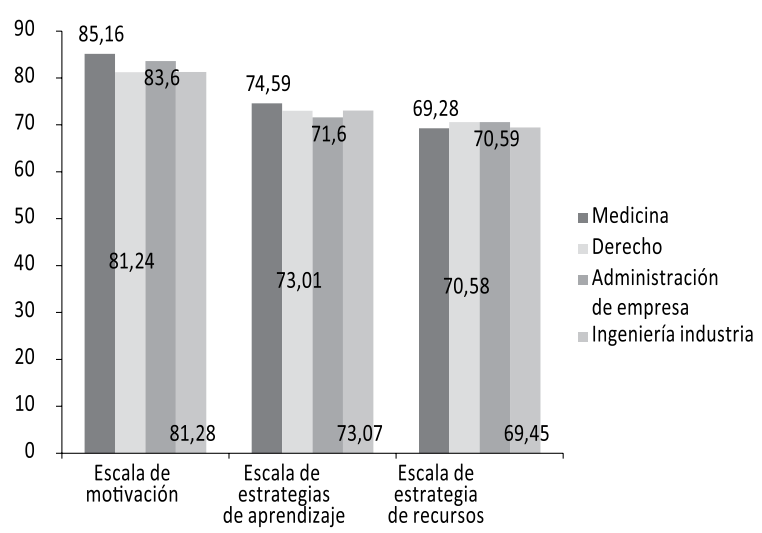

Figura 2. Resultados de estudiantes de programas académicos participantes en dimensiones evaluadas

Fuente: Resultado de investigación

La Figura 2 muestra el comportamiento de los programas en cada una de las escalas de manera general. Como resultado se observa que el Programa de Medicina en la escala de motivación obtuvo un porcentaje de 85,16\%, superior a los demás programas, mientras el Programa de
Derecho obtuvo el menor puntaje con un porcentaje de $81,24 \%$.

En la escala de estrategias de aprendizaje, el Programa de Medicina obtuvo un 74,59 \%, mientras que el Programa de Administración de Empresas obtuvo un 71,60\%, puntaje inferior en comparación con los resultados registrados por los demás programas evaluados.

En la escala de estrategia de recursos se muestra que el Programa de Administración de Empresas obtiene una puntuación de 70,59 \%, mientras que el Programa de Medicina obtuvo una puntuación de 69,28 \%, la cual es la menor en comparación con los demás programas evaluados.

La Figura 3 representa gráficamente los resultados obtenidos de manera general en la escala de autorregulación.

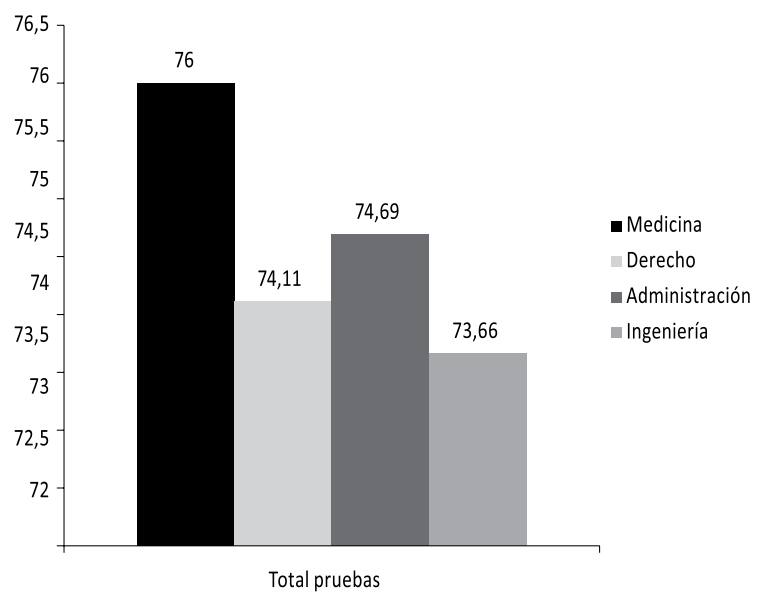

Figura 3. Resultados totales del MSLQ por programas

Fuente: Resultado de investigación 
Al finalizar el proceso de evaluación podemos observar que el Programa de Medicina obtuvo a nivel global en la prueba un 76,00\%, seguido de Administración de Empresas con un 74,69 \%. El Programa de Derecho se ubicó en un tercer lugar con un 74,11\%. Por su parte, el Programa de Ingeniera Industrial obtuvo una puntuación de 73,66\%, la menor en comparación con los demás programas evaluados.

En la subescala de ansiedad, el programa en el que los estudiantes manifiestan mayor grado de ansiedad fue Medicina con un 62,66 \%, mientras que los estudiantes del Programa de Ingeniería Industrial manejan un menor grado de ansiedad, el cual se vio reflejado con un promedio de $53,76 \%$.

\section{Discusión}

Revisando la literatura sobre el tema, se encuentran pocos estudios que realicen una comparación de las estrategias de autorregulación que utilizan los estudiantes según el programa académico cursado, aun cuando sí existen estudios descriptivos de un programa académico en particular. En ese sentido, este estudio muestra algunas consideraciones teniendo en cuenta las diferencias que se dieron en los estudiantes de los programas académicos que se tomaron como muestra.

A nivel general los resultados arrojados por la prueba nos indican que los estudiantes del Programa de Medicina obtuvieron el nivel más alto de autorregulación, seguido por los estudiantes del Programa de Administración de Empresas, mientras que los estudiantes de los Programas de Derecho e Ingeniería Industrial obtuvieron un nivel medio de autorregulación. Sin embargo, podemos resaltar que todos los estudiantes puntuaron por encima de la media, lo que indica un buen nivel de autorregulación, que muestra la existencia en los estudiantes de un proceso activo y constructivo a través del cual estos establecen metas para su aprendizaje mientras tratan de supervisar, regular y controlar su cognición, motivación y comportamientos dirigidos y limitados por metas y por características contextuales del entorno, según lo afirman Pintrich \& García (1993).

Con relación al primer objetivo orientado a identificar la motivación hacia el aprendizaje se encontró que todos los estudiantes de los programas evaluados obtuvieron resultados en un nivel alto tanto en la motivación intrínseca como extrínseca, en donde la primera está vinculada con aquellas acciones realizadas por el interés que genera la propia actividad, considerada como un fin en sí misma y no como un medio para alcanzar otras metas. Así mismo, la orientación motivacional extrínseca, se caracteriza generalmente como aquella que lleva al individuo a realizar una determinada acción para satisfacer otros motivos que no están relacionados con la actividad en sí misma, sino más bien con la consecución de otras metas que en el campo escolar suelen fijarse en obtener buenas notas, lograr reconocimiento por parte de los demás, evitar el fraca- 
so o ganar recompensas. Este resultado difiere al encontrado por Rinaudo, Chiecher y Donolo (2003) en una población de universitarios argentinos, que demostraron un nivel medio alto de motivación, donde la motivación intrínseca es mayor que la extrínseca.

Con respecto al segundo objetivo, orientado a la descripción de las estrategias de aprendizaje, los estudiantes de Medicina obtuvieron el nivel más alto seguido por los Programas de Administración de Empresas, Derecho e Ingeniería Industrial ubicados en un nivel medio. Esto puede indicar que los estudiantes de Medicina utilizan de forma recurrente estrategias de aprendizaje, que son aquellas acciones y pensamientos que ocurren durante el proceso de aprendizaje e influyen en la adquisición, retención y transferencia del conocimiento de una mejor manera que los alumnos de otros programas académicos, tal vez por la complejidad de su disciplina que los lleva a recurrir a diversas estrategias para asimilar los conocimientos de una forma eficaz y poder lograr sus metas educativas. Estos resultados confirman el estudio realizado por Salmerón, Gutiérrez-Braojos, Salmerón-Vilchez, y Rodríguez (2011) en una muestra de estudiantes universitarios españoles en la que se evidenció que las carreras del área de las Ciencias de la Salud presentan puntuaciones más altas en metas de logro y estrategias de autorregulación. Sin embargo, en ese mismo estudio los estudiantes de Ingeniería están en el mismo nivel que los de Medicina, lo cual no se corrobora en este estudio, donde obtienen puntajes medios en esta dimensión.
Sobre el tercer objetivo, relacionado con la identificación de las estrategias de manejo de los recursos, se encontró que todos los programas poseen un nivel medio; aquí se puede evidenciar que este es el aspecto que genera en ellos mayores dificultades, ya que ninguno alcanzó un nivel alto. Igualmente, contrario a la dimensión anterior, llama la atención que en esta dimensión el Programa de Medicina obtuvo menor puntuación en comparación con los datos arrojados en la evaluación de las otras dimensiones. En este aspecto los resultados demostraron que los estudiantes utilizan moderadamente las estrategias de tiempo y ambiente, programando y planeando los momentos de estudio solo en algunas ocasiones, en especial los del Programa de Medicina, cuyo menor porcentaje indica que necesitan optimizar su manejo del tiempo y el ambiente de estudio en el que frecuentemente se desenvuelven si quieren obtener mejores resultados académicos.

En cuanto a la ansiedad, como es sabido, consiste en la excesiva preocupación por el desempeño, sobre la cual los estudiantes mostraron un nivel medio, índice que puede afectar en algunas ocasiones su rendimiento, y que se correlaciona negativamente con el uso de estrategias de aprendizaje (Pintrich \& García, 1993). En este caso se observa que el Programa de Medicina presenta una escala de ansiedad mayor con respecto a los otros programas, tal vez ocasionado por las exigencias del pensum de estudio y la necesidad de optimizar la dimensión de estrategias de recursos para tener menores 
niveles de ansiedad. El Programa de Ingeniería Industrial presenta niveles de ansiedad menores que los otros programas.

De forma particular se evidencia cada uno de los resultados obtenidos en las subescalas evaluadas por el cuestionario, iniciando con la dimensión de motivación hacia el aprendizaje, dentro de la cual hacen parte los componentes de valor que se encuentran integrados por la orientación hacia la meta intrínseca, la orientación hacia la meta extrínseca y el valor a la tarea. A partir de esto podemos observar que el Programa de Medicina obtuvo una puntuación significativamente alta en la orientación hacia la meta intrínseca, a pesar que los demás programas también puntuaron dentro de un nivel alto. Ello sugiere que estos estudiantes se encuentran motivados a realizar aquellas acciones que les generan interés, considerándolas como un fin y no como un medio para alcanzar otras metas. Este resultado puede estar asociado al interés vocacional que suele identificar a los estudiantes de Ciencias de la Salud. Esta misma discusión fue planteada por Salmerón et al. (2011), quienes afirman que los estudiantes de las Ciencias de la Salud suelen cursar carreras que eligieron en primera opción, es decir, que realmente se inscribieron y fueron seleccionados en la carrera que deseaban cursar.

En general todos los programas mostraron una mayor orientación hacia la meta extrínse$c a$, la cual guía a los estudiantes a realizar una determinada acción para satisfacer otros motivos que no están relacionados con la actividad en sí misma, sino más bien con la consecución de otras metas que pueden referirse a la obtención de buenas notas, reconocimientos por parte de los demás o ganar recompensas. Así mismo la valoración positiva de las tareas representa un aspecto importante que le permite al estudiante involucrarse más en el propio aprendizaje y a utilizar estrategias cognitivas más frecuentemente, lo cual está evidenciando teóricamente una coherencia entre los resultados obtenidos, pues los estudiantes evaluados mostraron un nivel alto.

En la segunda dimensión, las estrategias cognitivas y metacognitivas, a su vez, se encuentran integradas por ensayo, elaboración, organización, pensamiento crítico y autorregulación metacognitiva. En cuanto al ensayo, los Programas de Medicina y Administración de Empresas arrojaron un nivel alto, mientras que los Programas de Derecho e Ingeniería Industrial mostraron un nivel bajo. Esto demuestra que el nivel de incidencia del ensayo en relación con la atención y los procesos de codificación en los estudiantes corresponde a una estrategia superficial de procesamiento de la información, que no ayuda a construir propiamente conexiones internas o integraciones de nueva información con el conocimiento previo, por lo que no es una muy buena estrategia de aprendizaje. Por su parte, en contraposición a esto, la elaboración y la organización permiten procesamientos más profundos de los materiales de estudio; de esta manera se encontró que todos los programas evaluados utilizan las dos últimas estrategias en un nivel alto a excepción del Programa de Ingeniería In- 
dustrial en lo que respecta a la estrategia de organización.

A pesar de que los programas evaluados en su mayoría arrojaron un nivel alto en las dos estrategias antes mencionadas, no se observa una congruencia con los resultados obtenidos en la estrategia de pensamiento crítico ni en la de autorregulación metacognitiva, pues todos los programas evaluados mostraron un nivel medio en la utilización de estas, lo cual significa que solo en algunas ocasiones los estudiantes utilizan el pensamiento crítico como un intento de pensar de un modo más profundo, reflexivo y crítico sobre el material de estudio (Pintrich et al., 1991). Así mismo las estrategias metacognitivas sugieren la utilización de tres procesos generales: el planeamiento, el control y la regulación, en donde se sugiere que el planear las actividades contribuye para activar aspectos relevantes del conocimiento previo que permiten organizar y comprender más fácilmente el material, al controlar las actividades se evalúa la atención y se cuestiona durante la lectura, en tanto que la regulación de las actividades refiere el continuo ajuste de las acciones cognitivas que se realizan en función del control previo. Un estudio realizado por Daura (2015) encontró que los estudiantes de Medicina obtienen puntajes altos en la escala de control, lo que les permite mayor dominio sobre el proceso de aprendizaje.

Por último, la tercera dimensión, que se refiere a la estrategia de manejo de los recursos, está integrada por tiempo y ambiente de estudio, regulación del esfuerzo, aprendizaje en pareja y buscar ayuda. Respecto a la estrategia de manejo de los recursos, todos los programas evaluados demostraron un nivel medio, lo cual significa que solo en algunas ocasiones programan y planean los momentos de estudio determinando específicamente un lugar de trabajo. Idealmente, el ambiente de estudio debe ser tranquilo, ordenado y relativamente libre de distractores visuales o auditivos (Pintrich, Smith, García \& Mckeachie, 1991). Este resultado se relaciona con el arrojado en la estrategia de regulación del esfuerzo, pues igualmente los estudiantes de los programas evaluados mostraron un nivel medio, es decir, que de alguna manera las habilidades de los estudiantes para persistir en las tareas, a pesar de las distracciones o falta de interés, puede deberse a que en algunos momentos no escogen los lugares adecuados para estudiar, asunto que influye de manera significativa en el éxito académico (Pintrich et al., 1991; Pintrich \& García, 1993).

Otras de las estrategias importantes en esta dimensión son el aprendizaje con pares y la búsqueda de ayuda, que aluden a la disposición de los estudiantes para plantear sus dificultades a un compañero o al docente. Según los datos obtenidos solo los estudiantes de Medicina evidenciaron en lo que se refiere al aprendizaje en pareja un nivel alto, mientras que los demás programas evaluados mostraron un nivel medio, tanto en el aprendizaje en pareja como en búsqueda de ayu$d a$, lo cual sugiere que solo en algunas ocasiones los estudiantes utilizan estas estrategias, pese a la relevancia de esta última, pues atiende al valor pedagógico que se atribuye al diálogo profesor- 
alumno y particularmente a los procesos de solicitar, dar y recibir ayuda pedagógica (Donolo, Rinaudo \& Chiecher, 2004).

Desde nuestra perspectiva como investigadoras podemos decir que los resultados muestran que muy probablemente los estudiantes que fueron objeto de análisis poseen una alta regulación en términos generales, ya que se encuentran niveles medios en algunas escalas. Este resultado contrasta con el estudio realizado por Allgood, Risko, Álvarez, y Fairbanks (2000), quienes, por el contrario, afirman que la mayoría de los estudiantes universitarios no son alumnos autorregulados. Por lo tanto, como investigadoras y críticas frente al proceso realizado se observa en la realidad que los docentes manifiestan su inquietud precisamente ante la falta de autonomía de los estudiantes en el proceso de aprendizaje, particularmente porque la universidad persigue en sus procesos de formación la búsqueda de estudiantes con pensamiento crítico, autorregulados, capaces de monitorear, planear y supervisar su proceso de aprendizaje de forma individual sin dejar de utilizar la herramienta de la comunicación bidireccional entre docente y estudiante.

Por esto es necesario continuar con el estudio de la autorregulación, desde otro tipo de estudios o metodologías, entre ellas las cualitativas, que corroboren los resultados obtenidos buscando espacios que promuevan el desarrollo de aspectos como el pensamiento crítico, la motivación tanto intrínseca como extrínseca y el manejo de los recursos que favorezcan el proceso de aprendizaje.
Cabe resaltar que este trabajo es útil tanto para el estudiante como para el docente, ya que los primeros se mostraron colaboradores e interesados en el tema, encontrándolo como un nuevo método que les ayudará al docente a tener una guía acerca de las herramientas que utilizan los estudiantes durante su proceso de aprendizaje, con la cual al docente se le facilitará también su proceso de enseñanza, según se vio reflejado en los comentarios recibidos por los estudiantes de los distintos programas evaluados. Por último, y con el fin de potencializar los resultados encontrados, el grupo investigador considera pertinente que los docentes se involucren en mejorar la autorregulación de sus estudiantes y se sugiere, como lo expone Coll (2014), replantear sus estrategias no solo pedagógicas, sino sus formas de evaluación, de tal manera, que estas se conviertan en un instrumento fundamental y útil para que el alumno pueda autorregular su propio proceso de aprendizaje a través del seguimiento, apoyo y tutoría que reciben de sus profesores.

\section{Referencias}

Allgood, W. P., Risko, V. J., Álvarez, M. C. \& Fairbanks, M. M. (2000). Factors that influence study. En R. F. Flippo \& D. C. Caverly (Coords.). Handbook of college reading and study strategy research (pp.201219). NJ: LEA.

Bandura, A. (1982). Teoría del aprendizaje social. Madrid: Espasa-Calpe.

Boekaerts, M. (1997). Self-regulated learning: A new concept embraced by researchers, policy makers, educators, teachers, and students. Learning and instruction, 7(2), 161-186. 
Coll, C. (2014). La evaluación continuada como instrumento para el ajuste de la ayuda pedagógica y la enseñanza de competencias de autorregulación. Cuaderno de Pedagogía Universitaria, 8(15), 14-20.

Daura, F. T. (2015). Aprendizaje autorregulado y rendimiento académico en estudiantes del ciclo clínico de la carrera de Medicina. Revista Electrónica de Investigación Educativa, 17(3), 28-45.

De la Fuente, J., Pichardo, M., Justicia, F. \& Berbén, A. (2008). Enfoques de aprendizaje, autorregulación y rendimiento en tres universidades europeas. Universidad de Alameira y Universidad de Granada. Psicothema, 20(4), 705-711. Recuperado desde: http://www.redalyc.org/articulo. oa? $\mathrm{id}=72720430$

Donolo, D., Rinaudo, M. \& Chiecher, A. (2004). Estudiantes, estrategias y contextos de aprendizaje presenciales y virtuales. Recuperado desde http://www. ateneonline.net/datos/22_02_Chiecher_ Anal\%C3\%ADa.pdf

Escorcia, D. (2010). Conocimientos metacognitivos y autorregulación: una lectura cualitativa del funcionamiento de los estudiantes universitarios en la producción de textos. Avances en Psicología Latinoamericana, 28(2), 265-277. Recuperado desde http://revistas.urosario.edu.co/index.php/ apl/article/view/1456/1328

García-Ros, R. \& Pérez González, F. (2011). Validez predictiva e incremental de las habilidades de autorregulación sobre el éxito académico en la universidad. Revista de Psicodidáctica/Journal of Psychodidactics, 16(2), 231-250. DOI: 10.1387/RevPsicodidact. 1002

Hernández Pina, F., Rosario, P., Cuesta Sáez de Tejada, J., Martínez Clares, P. \& Ruiz Lara, E. (2006). Promoción del aprendizaje estratégico y competencias de aprendizaje en estudiantes de primero de universidad: evaluación de una intervención. Revista de Investigación Educativa, 24(2), 615-631. Recuperado desde http:// revistas.um.es/rie/article/view/97221

Limón, M. (2004). Cambio conceptual y el aprendiz intencional esbozado por Paul R. Pintrich. Revista Electrónica de Investigación Psicoeducativa, 2(1), 175-184. Recuperado desde http://www.investigacion-psicopedagogica.org/revista/new/ ContadorArticulo.php?35

McCombs, B. (1989). Intervenciones educativas para potenciar la metacognición y el aprendizaje autorregulado. En J. Beltrán \& V. Bermejo (Comps.), Intervención Psicopedagógica. Madrid: Pirámide.

Núñez, J., Solano, P., Gonzales, J. C. \& Rosario, P. (2006). El aprendizaje autorregulado como medio y meta de la educación. Universidad de Oviedo, Universidad de Minho (Portugal). Papeles del Psicólogo, 27(3), 129-146.

Pintrich, P. \& García, T. (1993). Intraindividual differences in students' motivation and selfregulated learning. German Journal of Educational Psichology, 7(3), 99-107. 
Pintrich, P. R. \& García, T. (1993). Intraindividual differences in students' motivation and self-regulated learning. Zeitschrift für Pädagogische Psychologie/German Journal of Educational Psychology, 7(3), 99-107.

Pintrich, P., Smith, D., García, T. \& McKeachie, W. (1991). A Manual for the Use of the Motivational Strategies for Learning Questionnaire (MSLQ). Ann Arbor, MI: NCRIPTAL, The University of Michigan. Recuperado desde http://files.eric.ed.gov/ fulltext/ED338122.pdf

Pressley, M. \& Afflerbach, P. (1995). Verbal protocols of reading: The nature of constructively responsive reading. Hillsdale, NJ: Erlbaum.

Rinaudo, M. C., Chiecher, A. \& Donolo, D. (2003). Motivación y uso de estrategias en estudiantes universitarios. Su evaluación a partir del Motivated Strategies Learning Questionnaire. Anales de Psicología, 19(1), 107-119.

Rosário, P., Lourenço, A., Paiva, M. O., Núñez, J. C., González-Pienda, J. A. \& Valle, A. (2012). Autoeficacia y utilidad percibida como condiciones necesarias para un aprendizaje académico autorregulado. Anales de Psicología, 28(1), 37-44.

Salmerón, H., Gutiérrez-Braojos, C., SalmerónVilchez, P. \& Rodríguez, S. (2011). Metas de logro, estrategias de regulación y rendimiento académico en diferentes estudios universitarios. Revista de Investigación Educativa, 29(2), 467-486. Recuperado desde http://revistas.um.es/rie/ article/view/116041/135371
Sinatra, G. (2004). Contribuciones de Paul R. Pintrich a la investigación sobre cambio conceptual. Revista Electrónica de Investigación Psicoeducativa, 2(1), 171-174.

Tapia, J. A. (1995). Orientación educativa: Teoría, evaluación e intervención. Madrid: Síntesis.

Torrano, F. \& González, M. (2004). El aprendizaje autorregulado: presente y futuro de la investigación. Revista Electrónica de Investigación Psicoeducativa, 2(1), 1-34. Recuperado desde: http://www.investigacion-psicopedagogica.org/revista/articulos/3/espannol/Art_3_27.pdf

Valle, A., Cabanch, R., Rodríguez, S., Núñez, J. \& González, J. (2006). Metas académicas, estrategias cognitivas y estrategias de autorregulación del estudio. Psicothema, 18(2), 165-170.

Wood, R. \& Bandura, A. (1989). Impact of conceptions of ability on self-regulatory mechanisms and complex decision making. Journal of personality and social psychology, 56(3), 407-415.

Zimmerman, B. J. (1994). Dimensions of academic self-regulation: A conceptual framework for education. In D. H. Schunk \& B. J. Zimmerman (Eds.), Self-regulation of learning and performance: Issues and educational applications (pp.3-21). Hillsdale, NJ: Lawrence Erlbaum Associates, Inc.

Zimmerman, B. J. (1995). Self-regulation involves more than metacognition: A social cognitive perspective. Educational psychologist, 30(4), 217-221. 